\title{
Recurrent Giant Phyllodes Tumour in a Young Female: A Case Report
}

\author{
Mahim Koshariya ${ }^{1}$, Ashish Sharma ${ }^{1}$, Ajay Gehlot ${ }^{1}$, Surbhi Garg ${ }^{1}$, M.C. Songra ${ }^{1}$, Karan Peepre
}

Corresponding author:

Mahim Koshariya, MS, FMAS, FIASGO

Department of Surgery,

Gandhi Medical College \& Associated

Hamidia Hospital, Bhopal, India

E-mail: mahimk2000@yahoo.co.uk
'Department of Surgery; '2Department of Nuclear Medicine; Gandhi Medical College \& Associated Hamidia Hospital, Bhopal, India

\section{ABSTRACT}

Phyllodes tumors (PTs) are uncommon neoplasms of the breast, constituting 0.3 to $0.9 \%$ of all breast tumors in females comprised of both stromal and epithelial elements. The term cystosarcoma phyllode was coined by Johannes Muller, a misleading term as tumors are rarely cystic and the majority follow a benign clinical course. The term giant phyllodes is used when the tumor size exceeds $10 \mathrm{~cm}$ in diameter. WHO (World Health Organization) classification has identified three categories of phyllodes tumors of the breast (PT): benign, borderline and malignant. The mainstay of treatment of non-metastatic phyllodes tumors of the breast is complete surgical resection with wide resection margins. Local recurrence up to $50 \%$ after surgery has been reported in Phyllodes tumors. We report a case of two episodes of recurrent phyllodes tumor in a young female.

Key words: Phyllodes Tumors (PTs), recurrence, mastectomy

\section{INTRODUCTION}

The Phyllodes tumors (PTs) tumors of the breast are fibroepithelial tumors which are rarely seen and have potential for recurrence. Less than $1 \%$ of all the breast tumors consist of Phyllodes tumors (1-2). The majority of PTs occur in women between the age of 35 and 55 (3), although there are reported cases in adolescents, as well as in elderly women (4).

Phyllodes tumors are usually not considered initially in clinical diagnosis, show slow or rapid growth pattern, and are usually diagnosed after biopsy. These infrequent tumors are typically seen as a firm, circular, mobile, well-defined and painless mass in the diameter of 5 centimeters or more. The term giant phyllodes is used when the tumor size exceeds $10 \mathrm{~cm}$ in maximum diameter (4). PTs with diameters of 40 centimeters have also been reported in the literature (5). These lesions are difficult, if not impossible, to distinguish from fibroadenoma on physical examination or by radiologic studies except when 
quite large. The tumors are characterized by a combination of a hypercellular stroma and cleft like or cystic spaces lined by epithelium into which classically projects the stroma in a leaf-like fashion $(6,7)$. PTs are distinguished from FA histopathologically by their cellular pattern, having increased cellular atypical changes and excessive stromal growth. WHO (World Health Organization) classification has identified three categories of phyllodes tumors of the breast (PT): benign, borderline and malignant (8). Zurrida and his co-authors (9) reported that $9 \%, 44 \%$ and $33 \%$ of benign, borderline and malignant PTs developed local recurrence

\section{CASE REPORT}

A 32 year old lady presented to our department with chief complaint of lump in her right breast since 1 year. Patient had history of similar lump in past, 3 years back for which she was operated upon, a wide local excision was done and histopatjology report was consistent with phyllodes tumor, but it was followed by recurrence. Patient was again taken for surgery 2 years back, this time biopsy was indicative of Pericanalicular fibroadenoma. Unfortunately patient met with second time recurrence and patient was referred to us.

Examination revealed a large mass in the right breast measuring $18 \times 15 \mathrm{cms}$ with lobulated surface. Few dilated veins were noticed on the skin surface. The nipple was pushed down inferolaterally. Two scar marks of previous surgery were seen in inferomedial and outer upper quadrant (fig. $1 a, b)$. Contra lateral breast examination was normal, and there was no lymphadenopathy on bilateral axillary examination.

Mammography was done which showed recurrence of phyllode like growth in right breast with increased intralesional vascularity. USG breast showed large multilobulated mass lesion completely replacing right breast with very less fibrofatty glandular tissue, there was no significant axillary lymphadenopathy noted. USG abdomen was found to be normal. SPECT CT-Thorax and abdomen (fig. $2 a, b$ ) showed a mass lesion of $15 \mathrm{X} 12 \mathrm{~cm}$ with increased tracer uptake, no evidence of axillary or pectoral lymph node enlargement. There was no evidence of liver metastasis.

Bone scan (fig. 3) was found to be normal. FNAC done from the lump confirmed the diagnosis of phyllodes tumor.

The patient underwent surgical management. A simple mastectomy was performed with primary closure of the skin, without any skin graft or flap reconstruction. She had an uneventful post- operative course

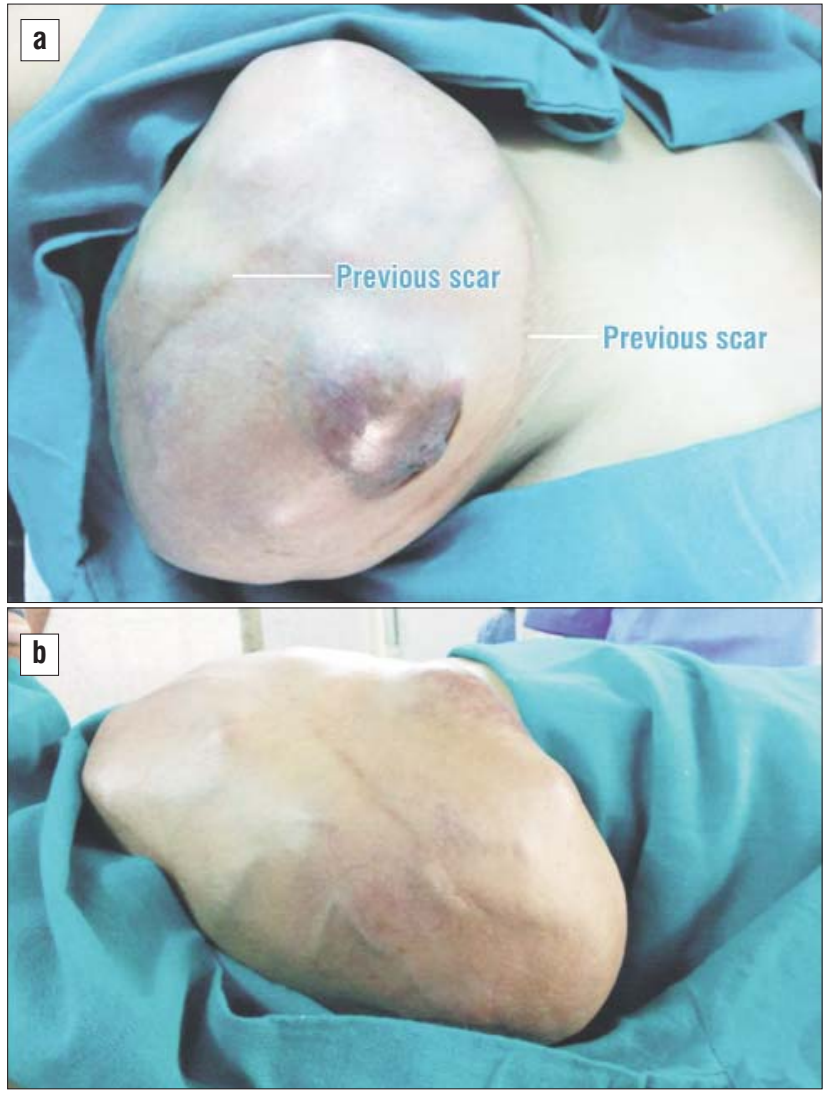

Figure 1 - (a) Gross appearance (anterior view) showing a large mass in right breast involving whole of the breast. The nipple was pushed down inferolaterally. Two scar marks of previous surgery were seen in inferomedial and outer upper quadrant

(b) Gross appearance (lateral view) showing large mass in the right breast measuring $18 \times 15 \mathrm{cms}$ with lobulated surface. Few dilated veins were also noticed

and was discharged on $8^{\text {th }}$ postoperative day. Cut section of mastectomy specimen revealed oval grey white tissue measuring $18 \times 15 \times 10 \mathrm{cms}$. Histological sections (fig. 6) revealed breast tissue showing slit like ducts with epithelial hyperplasia with cellular spindle stroma, no atypia mitosis or necrosis was seen in stroma, no sarcomatous areas seen, picture was suggestive of Benign Phyllodes tumor. Reconstructive breast surgery was offered to the patient but the patient refused surgery probably because there was history of two times recurrence. Patient was kept in regular follow up and after 1 year of surgery there was no evidence of local recurrence.

\section{DISCUSSION}

Tumors of mixed connective tissue and epithelium constitute an important group of unusual primary breast tumors. Phyllodes tumors contain a biphasic proliferation of mammary epithelium and stroma, the 


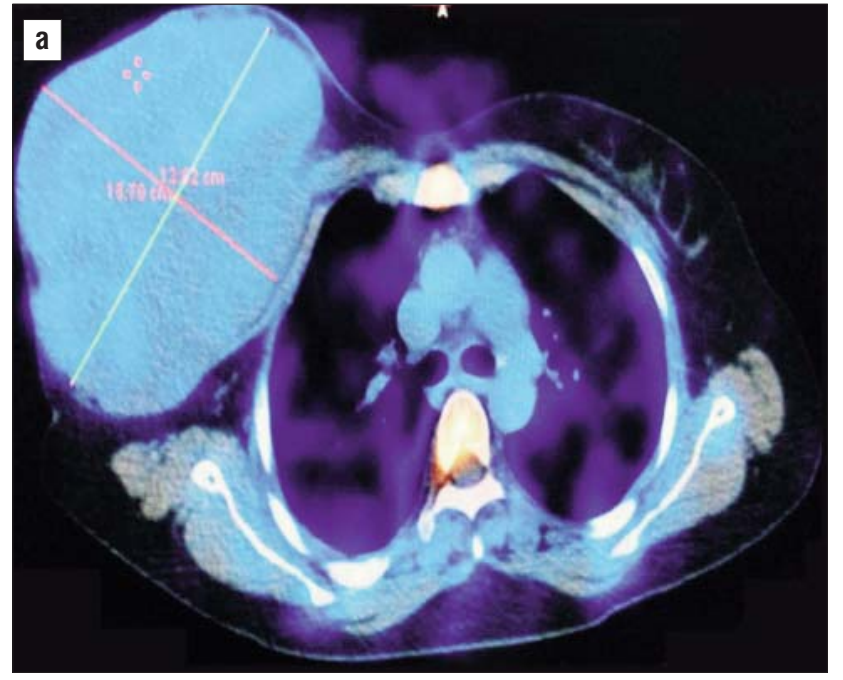

Figure 2 - (a) SPECT CT- (Transaxial view) showed a mass lesion of 15X $12 \mathrm{~cm}$ with increased tracer uptake, no evidence of axillary or pectoral lymph node enlargement. There was no evidence of liver metastasis. (b) SPECT CT- (Coronal View)

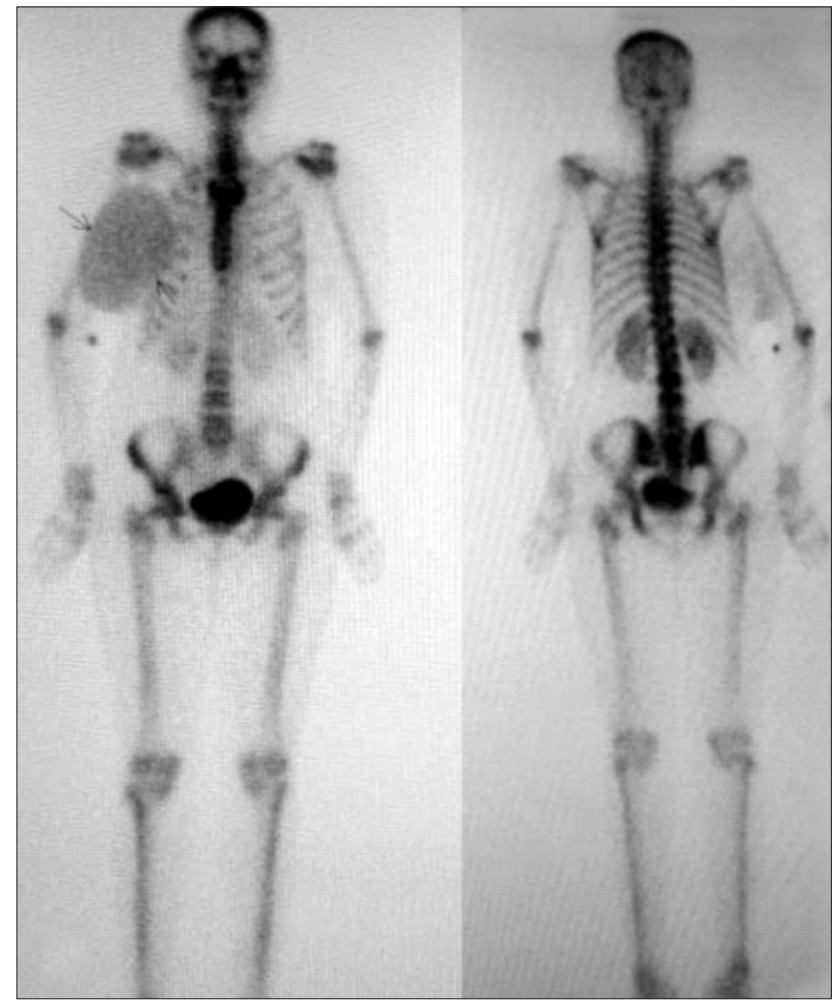

Figure 3 - Bone Scan- No abnormal uptake in skeleton reflects normal bone scan

latter determines the malignancy. Initially called cystosarcoma phylloides, a misleading description as tumors are rarely cystic and the majority follow a benign clinical course. With increasing cellularity, invasive margin and sarcomatous appearance, these tumors may be classified as malignant phyllodes
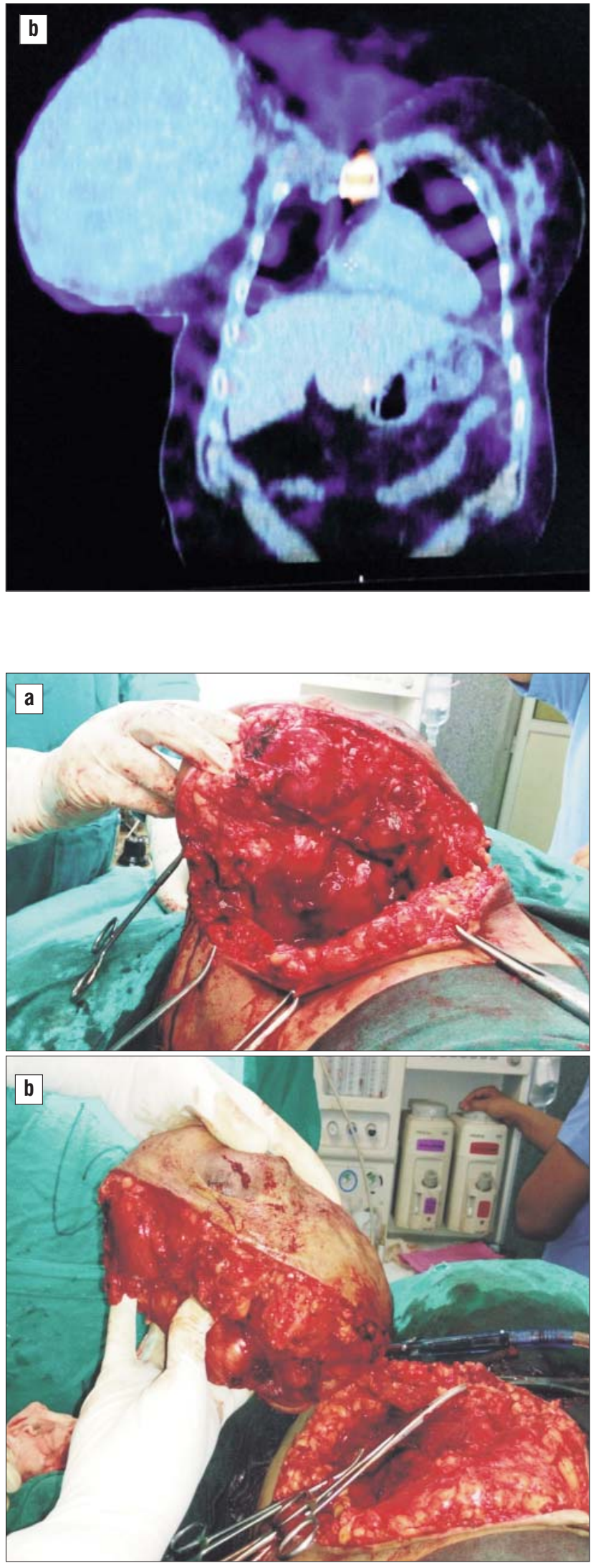

Figure 4 - (a) Intra-op picture showing dissection of tumor from chest wall. (b). Intra-op picture showing separation of tumor from chest wall 


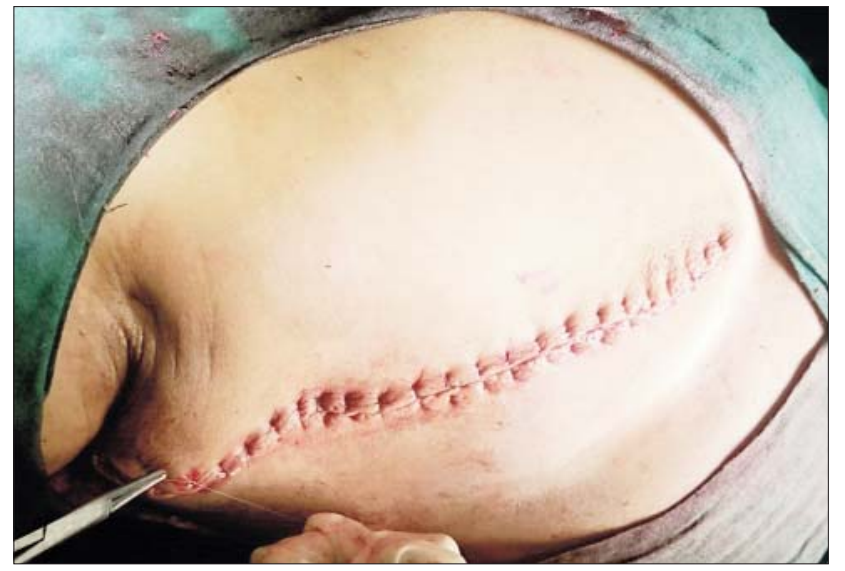

Figure 5 - Showing primary skin closure

tumors. Histologically, these tumors are similar to fibroadenomas, but the whorled stroma forms larger clefts lined by epithelium that resemble clusters of leaf like structures (10).

The majority of PTs occur in women between the age of 35 and 55 (3). Patients usually presents with complaints of a firm round macrolobulated mass which is painless in nature. Large lesions may be associated with dilated veins visible over the skin, which may be stretched and attenuated. Rare findings which have been reported are Nipple retraction $(4,11)$, skin ulceration invasion of the chest wall $(4,12,13)$, and bloody nipple discharge (13). Axillary lymph nodes are usually not involved however reactionary inflammatory lymph nodes are seen in about $20 \%$ cases (14)

Surgery remains the mainstay of treatment however the extent of surgical approach historically has been controversial, and continues to evolve. Complete surgical resection with wide resection margins (at least 1 $\mathrm{cm}$ ) should be performed, except for lesions larger than $10 \mathrm{~cm}$ in which total mastectomy is recommended. If the initial surgery fails to achieve a negative margin, it is recommended that the patient should undergo reexcision to obtain a wider margin in order to prevent local recurrence.

Mastectomy is advocated for large tumors for a microscopic margin of one centimeter without significant deformation of the breast, or recurrent tumors despite adequate margins (16). Axillary dissection is not routinely recommend as metastasis to the lymph nodes occur in less than $5 \%$ of patients

Mastectomy was the preferred treatment of choice in our case as the tumor was two times recurrent having a very large size covering almost whole of the breast. Mastectomy has been the most commonly performed surgery for borderline and malignant PTs
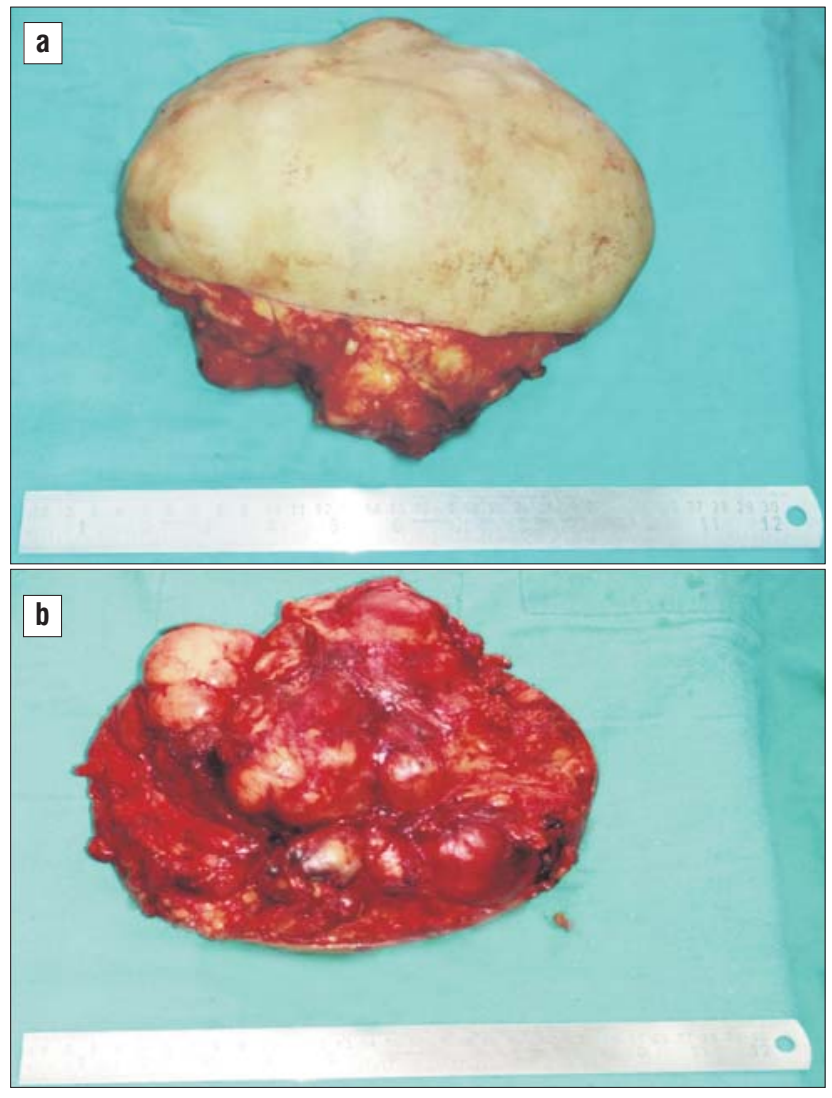

Figure 6 - (a) Resected specimen (gross appearance) showing a large mass with nodular surface and dilated veins.

(b) Cut section showing multilobulated surface

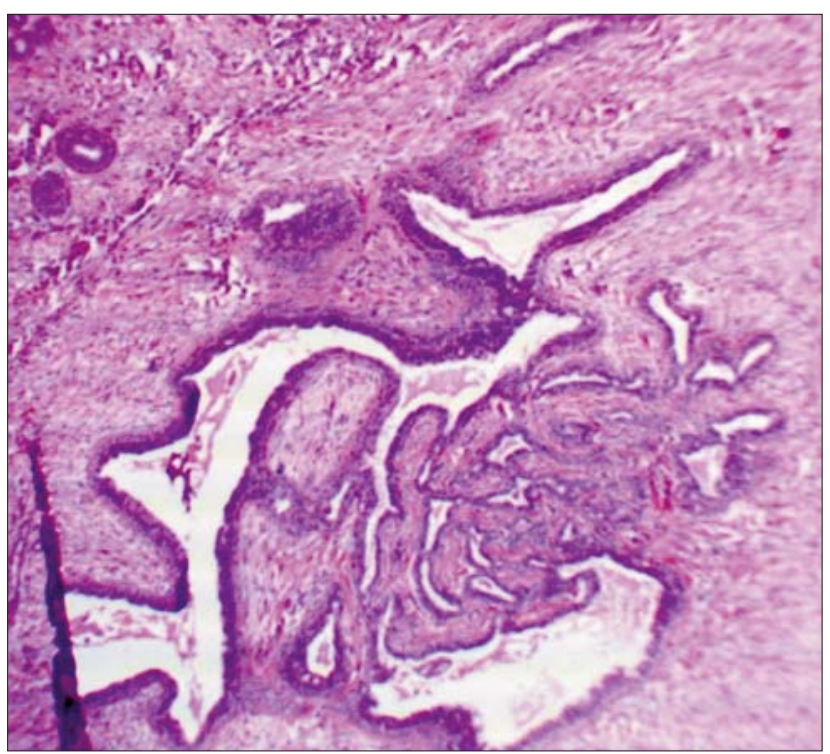

Figure 7 - Histopathological picture showing slit like ducts with epithelial hyperplasia with cellular spindle stroma, no atypia mitosis or necrosis seen in stroma, suggestive of benign phyllodes tumor 
with local recurrence rates of $5 \%$ and $12 \%$; respectively $(15,16,17)$.

Local recurrence is usually seen within the first few years of surgery and histologically resembles the original tumor. Occasionally, recurrent tumors show increased cellularity and more aggressive histological features than the original lesion. In most patients, local recurrence is isolated and is not associated with the development of distant metastasis (18). Important risk factors predicting recurrence is positive margin, young age at presentation, large tumor size, histological type. Other parameters such as stromal hypercellularity, stromal atypia, stromal overgrowth, and mitosis, were significantly associated with local recurrence (19). The most important risk factor for recurrence is the resection done within $1-2 \mathrm{~cm}$ negative surgical margins (20). It is important that these factors be identified and appropriate surgical procedure be selected to ensure clear margins.

Radiotherapy has a role in reducing local recurrence in borderline and malignant phylloides but has no impact on over all survival and disease free survival (21).

\section{CONCLUSION}

Phyllodes tumor are rare tumors of breast but are notorious for local recurrence. Wide local excision is an acceptable method of treatment but for recurrent and large tumors $(>10 \mathrm{~cm})$ simple mastectomy is preferred for complete tumor excision. Most important factor to prevent local recurrence is to keep a sufficient margin of healthy tissue. It is necessary that the patients be kept in follow up as there is a risk of local recurrence and distant metastasis.

\section{REFERENCES}

1. Azzopardi JG. Sarcoma in the breast. In Benningron J (ed). Problems in Breast Pathology. Major Problems in Pathology. Vol 11, Philadelphia: WB Saunders Co; 1979. p. 335-359.

2. Rowell MD, Perry RR, Hsiu JG, Barranco SC. Phlyllodes tumors. Am J Surg. 1993 Mar;165(3):376-9.

3. Salvadori B, Cusumano F, Del-Bo R, Delledonne V, Grassi M, Rovini D, et al. Surgical treatment of phyllodes tumors of the breast. Cancer. 1989 Jun 15;63(12):2532-6.

4. Reinfuss M, Mituś J, Duda K, Stelmach A, Ryś J, Smolak K. The treatment and prognosis of patients with phyllodes tumour of the breast: an analysis of 170 cases. Cancer. 1996 Mar 1;77(5):9106.

5. Liang Ml, Ramaswamy B, Patterson CC, McKelvey MT, Gordillo G, Nuovo GJ, et al. Giant breast tumors: Surgical man agement of phyllodes tumors, potential for reconstructive sur gery and a review of literature. World J Surg Oncol. 2008;11:117.

6. Pietrusuzka M, Barnes L. Cystosarcoma phyllodes: a clinicopathologic analysis of 42 cases. Cancer. 1978 May;41(5):1974-83.

7. Moffat CJ, Pinder SE, Dixon AR, Elston CW, Blamey RW, Ellis I0. Phyllodes tumours of the breast: a clinicopathological review of thirty-two cases. Histopathology. 1995 Sep;27(3):205-18.

8. World Health Organization. Histological typing of breast tumors. Tumori. 1982 Jun 30;68(3):181-98.

9. Zurrida S, Bartoli C, Galimberti V, Squicciarini P, Delledonne V, Veronesi P. Which therapy for unexpected phyllode tumour of the breast? Eur J Cancer. 1992;28(2-3):654-7.

10. Kelly K. Hunt, Marjorie C. Green, and Thomas A. Buchholz. Diseases of the breast. In Sabiston textbook of surgery, Nineteenth ed USA: Elsevier-Saunders; 2012. p. 845.

11. Deodhar SD, Joshi S, Khubchandani S. Cystosarcoma phyllodes. J Postgrad Med. 1989 Apr;35(2):98-103.

12. Dyer NH, Bridger JE, Taylor RS. Cystosarcoma phylloides. Br J Surg. 1966 May;53(5):450-5.

13. Chen WH, Cheng SP, Tzen CY, Yang TL, Jeng KS, Liu CL, et al. Surgical treatment of phyllodes tumors of the breast: retrospective review of 172 cases. J Surg Oncol. 2005 Sep 1;91(3):185-94.

14. Rowell MD, Perry RR, Hsiu JG, Barranco SC. Phyllodes tumors. Am J Surg. 1993 Mar;165(3):376-9.

15. Treves N, Sunderland DA. Cystosarcoma phyllodes of the breast: a malignant and a benign tumor; a clinicopathological study of seventyseven cases. Cancer. 1951 Nov;4(6):1286-1332.

16. Asoglu 0, Mustafa M, Blanchard K, Grant CS, Reynolds C, Cha SC, et al. Risk factors for recurrence and death after primary surgical treatment of malignant phyllodes tumors. Ann Surg Oncol. 2004 Nov; 11(11):1011-7.

17. Barth RJ Jr. Histologic features predict local recurrence after breast conserving therapy of phyllodes tumors. Breast Cancer Res Treat. 1999 0ct;57(3):291-5.

18. Ahmad Z, Koshariya M, Shukla S, Vatti V, Diwan A. A rare case of recurrent malignant phyllodes tumor of the breast in a young nulliparous woman. Clin Cancer Investig J. 2014;3:173-5.

19. Wei J, Tan YT, Cai YC, Yuan ZY, Yang D, Wang SS, et al. Predictive factors for the local recurrence and distant metastasis of phyllodes tumors of the breast: a retrospective analysis of 192 cases at a single center. Chin J Cancer. 2014 0ct;33(10):492-500. doi: 10.5732/ cjc.014.10048. Epub 2014 Aug 8.

20. Lenhard MS, Kahlert S, Himsl I, Ditsch N, Untch M, Bauerfeind I. Phyllodes tumour of the breast: clinical follow-up of 33 cases of this rare disease. Eur J Obstet Gynecol Reprod Biol. 2008 Jun;138(2):21721. Epub 2007 Sep 14.

21. Zeng S, Zhang X, Yang D, Wang X, Ren G. Effects of adjuvant radiotherapy on borderline and malignant phyllodes tumors: a systematic review and meta analysis. Mol Clin Oncol. 2015 May;3(3):663-671. Epub 2015 Feb 6. 